Development and characterization of the superconducting integrated receiver channel of the TELIS atmospheric sounder

This article has been downloaded from IOPscience. Please scroll down to see the full text article.

2010 Supercond. Sci. Technol. 23045016

(http://iopscience.iop.org/0953-2048/23/4/045016)

The Table of Contents and more related content is available

Download details:

IP Address: 129.247.247.239

The article was downloaded on 01/04/2010 at 09:48

Please note that terms and conditions apply. 


\title{
Development and characterization of the superconducting integrated receiver channel of the TELIS atmospheric sounder
}

\author{
Gert de Lange ${ }^{1}$, Manfred Birk ${ }^{2}$, Dick Boersma ${ }^{1}$, \\ Johannes Dercksen ${ }^{1}$, Pavel Dmitriev ${ }^{3}$, Andrey B Ermakov ${ }^{1,3}$, \\ Lyudmila V Filippenko ${ }^{3}$, Hans Golstein ${ }^{1}$, Ruud W M Hoogeveen ${ }^{1}$, \\ Leo de Jong ${ }^{1}$, Andrey $\mathbf{V}$ Khudchenko ${ }^{1,3}$, Nickolay $\mathbf{V}$ Kinev $^{1,3}$, \\ Oleg S Kiselev ${ }^{1,3}$, Bart van Kuik ${ }^{1}$, Arno de Lange ${ }^{1}$, \\ Joris van Rantwijk ${ }^{1}$, Avri M Selig ${ }^{1}$, Alexander S Sobolev ${ }^{3}$, \\ Mikhail Yu Torgashin ${ }^{3}$, Ed de Vries ${ }^{1}$, Georg Wagner ${ }^{2}$, \\ Pavel A Yagoubov ${ }^{1,4}$ and Valery P Koshelets ${ }^{1,3}$ \\ ${ }^{1}$ SRON Netherlands Institute for Space Research, PO Box 800, 9700 AV Groningen, \\ The Netherlands \\ ${ }^{2}$ DLR German Aerospace Centre, Remote Sensing Technology Institute, D-82234 Wessling, \\ Germany \\ ${ }^{3}$ The Kotel'nikov Institute of Radio Engineering and Electronics, Russian Academy of \\ Science, 11/7 Mokhovaya Street, 125009, Moscow, Russia \\ ${ }^{4}$ European Organization for Astronomical Research in the Southern Hemisphere (ESO), \\ Karl-Schwarzschild-Strasse 2, D-85748 Garching bei München, Germany \\ E-mail: G.de.Lange@ sron.nl and valery@hitech.cplire.ru
}

Received 18 November 2009, in final form 20 January 2010

Published 10 March 2010

Online at stacks.iop.org/SUST/23/045016

\begin{abstract}
The balloon-borne instrument TELIS (TErahertz and submillimetre LImb Sounder) is a three-channel superconducting heterodyne spectrometer for atmospheric research use. It detects spectral emission lines of stratospheric trace gases that have their rotational transitions at $\mathrm{THz}$ frequencies. One of the channels is based on the superconducting integrated receiver (SIR) technology. We demonstrate for the first time the capabilities of the SIR technology for heterodyne spectroscopy in general, and atmospheric limb sounding in particular. We also show that the application of SIR technology is not limited to laboratory environments, but that it is well suited for remote operation under harsh environmental conditions. Within a SIR the main components needed for a superconducting heterodyne receiver such as a superconductor-insulator-superconductor (SIS) mixer with a quasi-optical antenna, a flux-flow oscillator (FFO) as the local oscillator, and a harmonic mixer to phase lock the FFO are integrated on a single chip. Light weight and low power consumption combined with broadband operation and nearly quantum limited sensitivity make the SIR a perfect candidate for use in future airborne and space-borne missions. The noise temperature of the SIR was measured to be as low as $120 \mathrm{~K}$, with an intermediate frequency band of $4-8 \mathrm{GHz}$ in double-sideband operation. The spectral resolution is well below $1 \mathrm{MHz}$, confirmed by our measurements. Remote control of the SIR under flight conditions has been demonstrated in a successful balloon flight in Kiruna, Sweden. The sensor and instrument design are presented, as well as the preliminary science results from the first flight.
\end{abstract}

(Some figures in this article are in colour only in the electronic version) 


\section{Introduction}

TELIS (TErahertz and submillimetre LImb sounder) [1-3] is a three-channel balloon-borne heterodyne spectrometer for atmospheric research developed in a collaboration of three institutes: Deutsches Zentrum für Luft- und Raumfahrt (DLR), Germany, Rutherford Appleton Laboratories (RAL), United Kingdom, and SRON-Netherlands Institute for Space Research, the Netherlands. The three receivers utilize stateof-the-art superconducting heterodyne technology and operate at $500 \mathrm{GHz}$ (by RAL), at $480-650 \mathrm{GHz}$ (by SRON in tight collaboration with Kotel'nikov Institute of Radio Engineering and Electronics, IREE, Moscow), and at $1.8 \mathrm{THz}$ (by DLR). TELIS is designed to be a compact, lightweight instrument capable of providing broad spectral coverage, high spectral resolution and long flight duration. The TELIS instrument serves also as a test bed for many novel cryogenic technologies and as a pathfinder for satellite based instrumentation.

TELIS is mounted on the same balloon platform as the Fourier transform spectrometer MIPAS-B [4], developed by IMK (Institute of Meteorology and Climate research of the University of Karlsruhe, Germany) and is operated in the mid-infrared (680-2400 $\left.\mathrm{cm}^{-1}\right)$. Both instruments observe simultaneously the same air mass, and together they yield an extensive set of stratospheric constituents that can be used for detailed analysis of atmospheric chemical models, such as ozone destruction cycles. In particular, the $480-650 \mathrm{GHz}$ TELIS channel is able to measure vertical profiles of $\mathrm{ClO}$, $\mathrm{BrO}, \mathrm{O}_{3}$ and its rare isotopologues, $\mathrm{O}_{2}, \mathrm{HCl}, \mathrm{HOCl}, \mathrm{H}_{2} \mathrm{O}$ and three rare isotopologues, $\mathrm{HO}_{2}, \mathrm{NO}, \mathrm{N}_{2} \mathrm{O}, \mathrm{NO}_{2}, \mathrm{HNO}_{3}$, $\mathrm{CH}_{3} \mathrm{Cl}$, and $\mathrm{HCN}$. In this paper the design and technology for the 480-650 GHz channel as used in the flight configuration are presented in conjunction with test results and the first preliminary scientific results.

\section{Superconducting integrated receiver design}

A superconducting integrated receiver (SIR) [5, 6] was proposed more than 10 years ago and has since then been developed up to the point of practical applications [7, 8]. A SIR comprises on one chip (size of $4 \mathrm{~mm} \times 4 \mathrm{~mm} \times 0.5 \mathrm{~mm}$ ) the key elements needed for heterodyne detection: a low-noise SIS mixer with quasi-optical antenna, a flux-flow oscillator (FFO) [9] acting as a local oscillator (LO) and a second SIS harmonic mixer (HM) for the FFO phase locking, see figure 1. The concept of the SIR is very attractive for many practical applications because of the compactness and the wide tuning range of the FFO [10]. Presently, the frequency range of most practical heterodyne receivers is limited by the tuning range of the local oscillator, typically $10-15 \%$ for a solid-state multiplier chain [11]. In the SIR the bandwidth is determined by the SIS mixer tuning structure and the matching circuitry between the SIS and the FFO. A bandwidth up to 30-40\% may be achieved with a twin-junction SIS mixer design. Another potential advantage is the use of arrays of SIR channels within a single cryostat that could operate at the same or different LO frequencies.

Whether the increase in sensitivity and compactness of the SIR counter balances the increase in complexity (especially

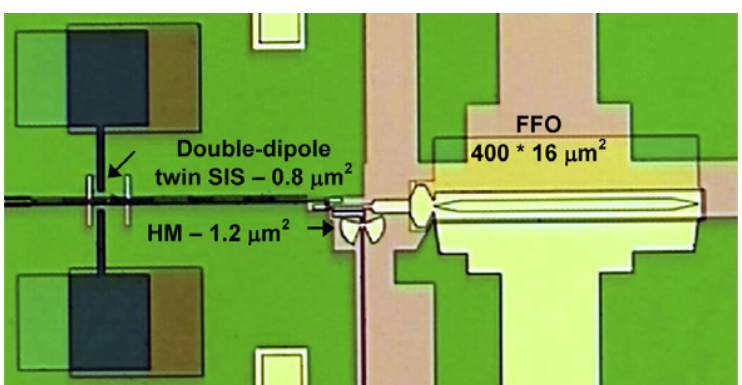

Figure 1. Central part of the SIR chip with the twin SIS mixer and double-dipole antenna on the left, the FFO on the right and the harmonic mixer for FFO phase locking in the middle.

the required He cooling) as compared to a room temperature Schottky receiver, depends on the scientific goals to be achieved. The enhanced sensitivity enables detection of trace gases with only small spectroscopic features. Also, short integration times allow for rapid limb scanning, which can lead to increased spatial resolution as was proposed for the future GACM mission [12].

All components of the SIR microcircuits are fabricated in a high quality $\mathrm{Nb}-\mathrm{AlN} / \mathrm{NbN}-\mathrm{Nb}$ tri-layer on a Si substrate [13]. The receiver chip is placed on the flat back surface of a silicon lens, forming an integrated lens-antenna. As the FFO is very sensitive for external electromagnetic interferences the SIR chip is shielded by two concentric cylinders: the outer cylinder is made of cryo-perm and the inner one of copper with a $100 \mu \mathrm{m}$ coating of superconducting lead.

The resolution of the TELIS back-end spectrometer is 2.160 MHz, sufficient to resolve the exact shape of atmospheric lines. The FFO line shape and spectral stability should ideally be much better than this. However, the free-running linewidth of the FFO can be up to $10 \mathrm{MHz}$ and therefore a phase-lock loop (PLL) has been developed to phase lock the FFO to an external reference oscillator $[6,14]$. For this, a small fraction of the FFO power is first directed to a so-called Harmonic Mixer (HM), placed on the SIR chip. The HM is pumped by an off-chip local oscillator source unit (LSU) which is a tunable reference frequency in the range of 19-21 GHz. The frequency of the LSU is chosen such that the difference frequency of the $n$th harmonic of the LSU, as generated by the HM, and the FFO is about $4 \mathrm{GHz}$. This difference signal is then amplified by a cryogenic low-noise HEMT amplifier and down-converted to $400 \mathrm{MHz}$ by using a second reference at $3.6 \mathrm{GHz}$. Finally, the frequency and phase of this $400 \mathrm{MHz}$ signal is compared against yet another reference frequency of $400 \mathrm{MHz}$ and the resulting error signal is fed back to the FFO. The LSU and the reference signals at $3.6 \mathrm{GHz}$ and at $400 \mathrm{MHz}$ are all phase locked to an internal ultrastable $10 \mathrm{MHz}$ Master oscillator.

\section{TELIS instrument design}

The front-end of the TELIS instrument is common for the three channels and consists of the pointing telescope, a calibration blackbody, relay and band-separating optics (see figure 2). Details of the optical design can be found in [15-17]. The three 


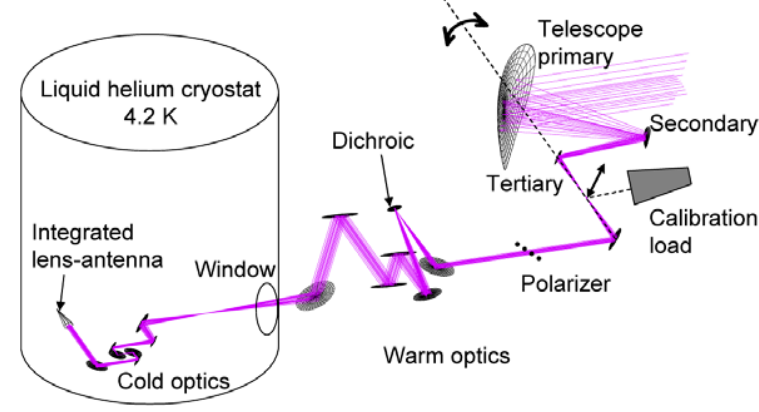

Figure 2. Optical lay-out of the TELIS-SIR channel.

mirrors of the dual offset Cassegrain telescope are mounted on a common frame, rotatable around the optical axis of the output beam. Limb scanning is performed between the upper troposphere $(8-10 \mathrm{~km}$ in the Arctic) to flight altitude (typically $32 \mathrm{~km}$ ) in 1-2 km steps. At the tangent point of the line of sight, the vertical (elevation) resolution is about $2 \mathrm{~km}$ for an observational frequency of $500 \mathrm{GHz}$, scaling inversely proportional with frequency. In horizontal (azimuth) direction the spatial resolution is about a factor of two less due to the anamorphicity of the telescope. This is allowed as the atmospheric properties within the beam hardly depend on the azimuth.

The radiometric gain of the spectrometers is calibrated once or twice in every Limb scan using a conical blackbody reference source and a measurement of the cold sky. For this, a small flip mirror is included between the telescope and the beam-separating optics. By measuring at two up-looking telescope positions, the impact of the remaining air above the gondola can be assessed.

Simultaneous observations by the receivers is achieved by quasi-optical beam splitting: first a wire-grid-based polarizing beam splitter is employed to reflect one linear polarization to the $500 \mathrm{GHz}$ channel, the other linear polarization is split by a dichroic filter between the SIR channel and the THz channel. Subsequently, offset mirrors shape and direct the three beams to the cryogenic channels. Inside the custom designed liquid-helium-cooled cryostat, each receiver has dedicated cold optics, a superconducting mixing element and intermediate frequency (IF) amplifiers.

The very compact $500 \mathrm{GHz}$ receiver channel consists of a fixed-tuned waveguide SIS mixer, a cryogenic solid-state local oscillator (LO) chain and a low-noise IF chain operating at a relatively high IF frequency (IF $=15-19 \mathrm{GHz}$ ) [18]. The $1.8 \mathrm{THz}$ channel employs a cryogenic solid-state LO that is lossless coupled into the mixer via a Martin-Puplett type optical interferometer. The mixer is based on a phonon-cooled NbN HEB (hot electron bolometer) [19]. The 480-650 GHz SIR receiver channel is based on a single-chip superconducting integrated receiver (SIR), as described in section 2.

The warm optics couples to the SIR channel with a beam that has a waist radius ranging from 2 to $3 \mathrm{~mm}$, located at the cryostat window. The system-pupil is imaged by two additional mirrors on the silicon elliptical lens; on the back surface of this lens the SIR chip is located. The SIR channel
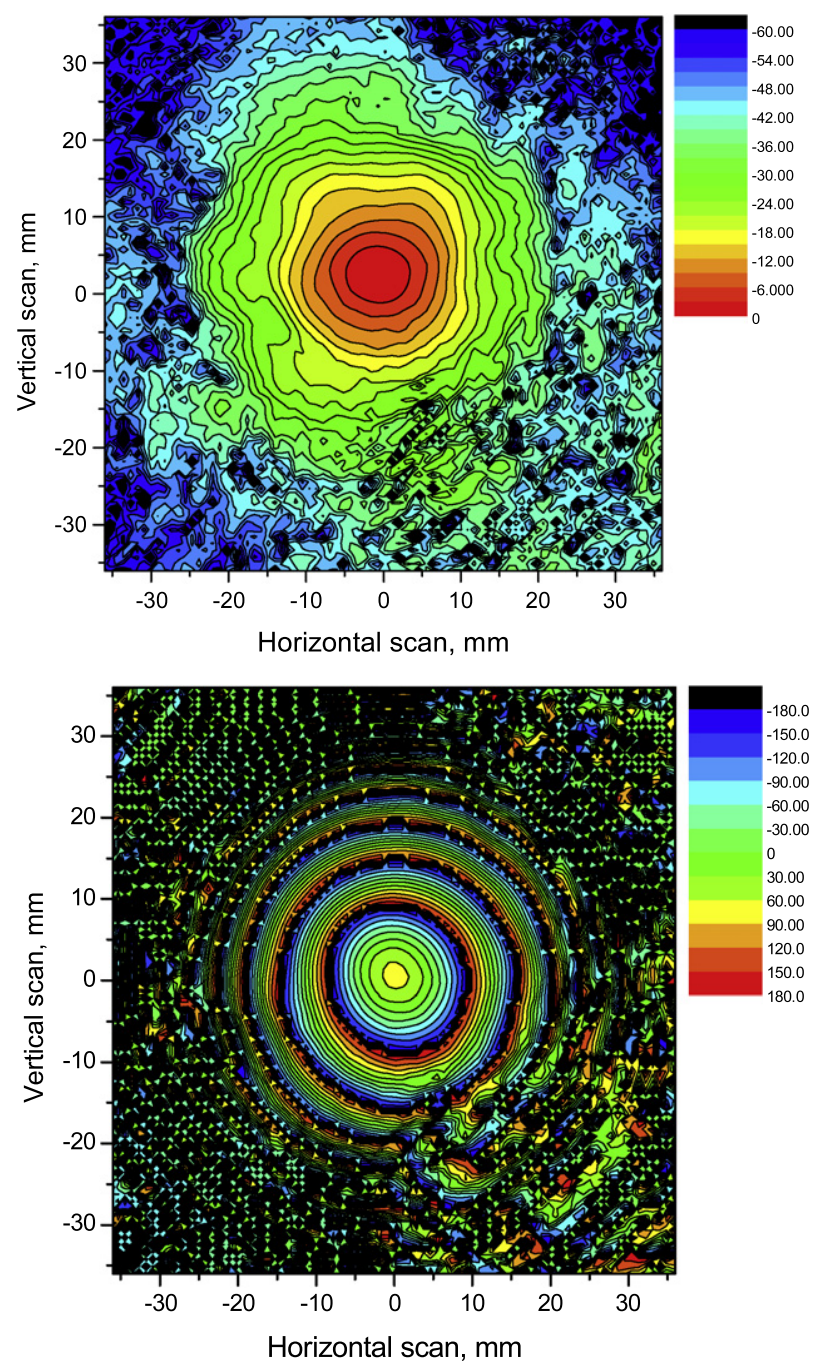

Figure 3. The amplitude (top figure) and phase (lower figure) distribution of the near field beam of the SIR channel. The amplitude is given in units of $\mathrm{dB}$. The distance from the beam waist is $110 \mathrm{~mm}$ and the frequency is $600 \mathrm{GHz}$ [3].

cold optics is also frequency independent to fully exploit the wide-band operation of the SIR device.

The amplitude-phase distribution of the near field beam of the SIR cold channel at $600 \mathrm{GHz}$ as measured at the dewar window is shown in figures 3(a) and (b). The beam waist is measured to be $2.25 \mathrm{~mm}$, which is within $1 \%$ of the designed value. The measured Gaussisity of the beam is $92.4 \%$.

The IF processor (located on the main frame of TELIS) converts the amplified IF output signals of the three receivers to the input frequency range of the digital autocorrelator. The digital autocorrelator has a bandwidth of $2 \mathrm{GHz} \times 2 \mathrm{GHz}$ with 2048 spectral channels. Both the IF processor and the digital autocorrelator are developed by Omnisys instruments AB [20].

The SIR channel is controlled with a battery-operated ultralow-noise biasing system. Since noise on the bias lines of the FFO translates in a wider FFO linewidth, several precautions, like decoupling of digital control lines and extensive filtering and shielding, are implemented. The SIR bias unit is digitally controlled by the on-board DLR PC-104 


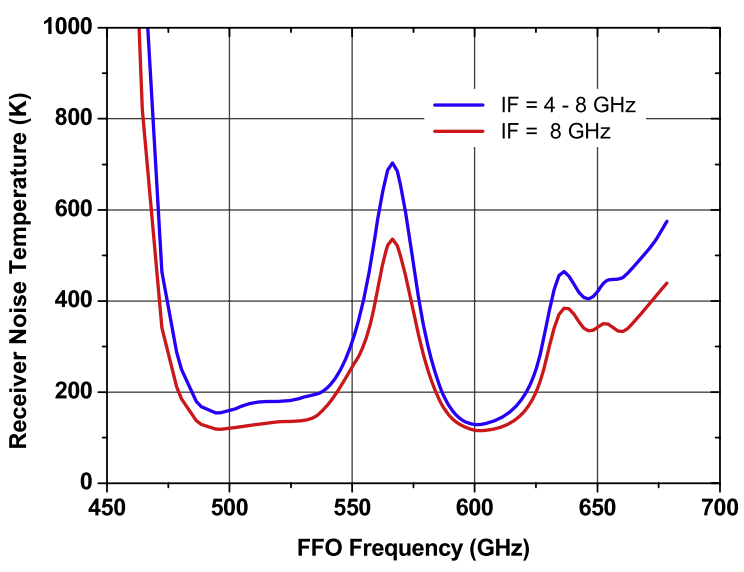

Figure 4. Measured DSB receiver noise temperature of the SIR device selected for flight at $8 \mathrm{GHz}$ IF frequency (red, lower curve) and integrated in the 4-8 GHz IF range (blue, upper curve).

computer, that also interfaces with the other channels, the digital autocorrelator, and with the host instrument MIPAS. A radio link provides real-time two-way contact with the ground segment consisting of a server computer with three dedicated client computers, coupled through TCP/IP socket connections. The complete system is dimensioned to have sufficient cooling liquids and battery power for a $24 \mathrm{~h}$ flight.

\section{TELIS-SIR channel performance}

The TELIS-SIR channel has been characterized in eight microwindows that have been selected for the flight in Kiruna. These micro-windows have the following LO frequencies:

- $495.04 \mathrm{GHz}$ for $\mathrm{H}_{2}{ }^{18} \mathrm{O}$;

- $496.88 \mathrm{GHz}$ for HDO;

- $505.60 \mathrm{GHz}$ for $\mathrm{BrO}$;

- $507.27 \mathrm{GHz}$ for $\mathrm{ClO}$;

- $515.25 \mathrm{GHz}$ for $\mathrm{O}_{2}$, pointing, and temperature;

- $519.25 \mathrm{GHz}$ for $\mathrm{BrO}$ and $\mathrm{NO}_{2}$;

- $607.70 \mathrm{GHz}$ for ozone isotopes;

- $619.10 \mathrm{GHz}$ for $\mathrm{HCl}, \mathrm{ClO}$ and $\mathrm{HOCl}$.

Initial flight values for the parameters for the FFO, SIS and HM mixers have been determined for each micro-window. Dedicated algorithms allowing for fast switching between LO frequencies and for in-flight optimization of the SIR, have been developed (see below). It takes about 1 min of stabilization and optimization to switch between two LO settings. All experimental results discussed here have been obtained with the SIR flight device.

The measured double-sideband (DSB) receiver noise temperature $T_{\mathrm{R}}$, uncorrected for any loss, is presented in figure 4 as a function of LO frequency, and in figure 5 as a function of IF frequency. As can be seen, the noise is well below $200 \mathrm{~K}$ at all frequencies of interest, with a minimum of $120 \mathrm{~K}$ at 500 and $600 \mathrm{GHz}$. The noise peak around 540$575 \mathrm{GHz}$ is partially spurious, caused by absorption of water vapour in the path between calibration sources and the cryostat, and partially real due to properties of the SIS mixer tuning

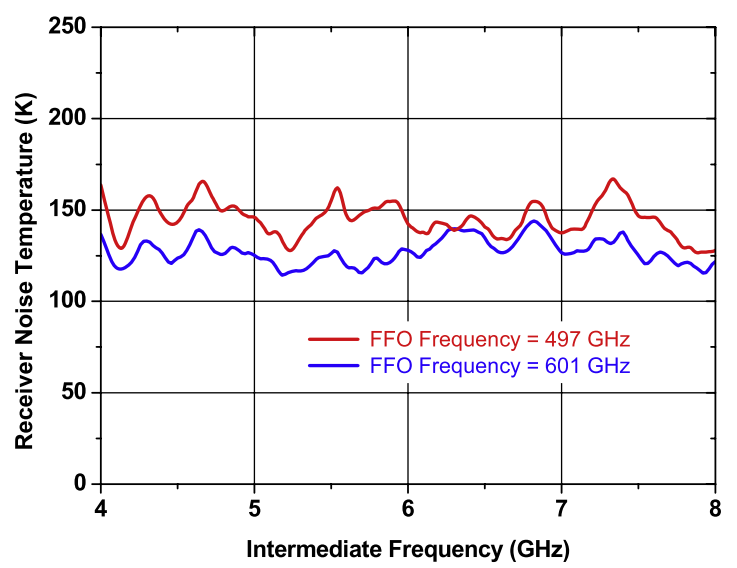

Figure 5. Receiver noise temperature as a function of IF frequency, taken at two FFO frequencies: 497 and $601 \mathrm{GHz}$.

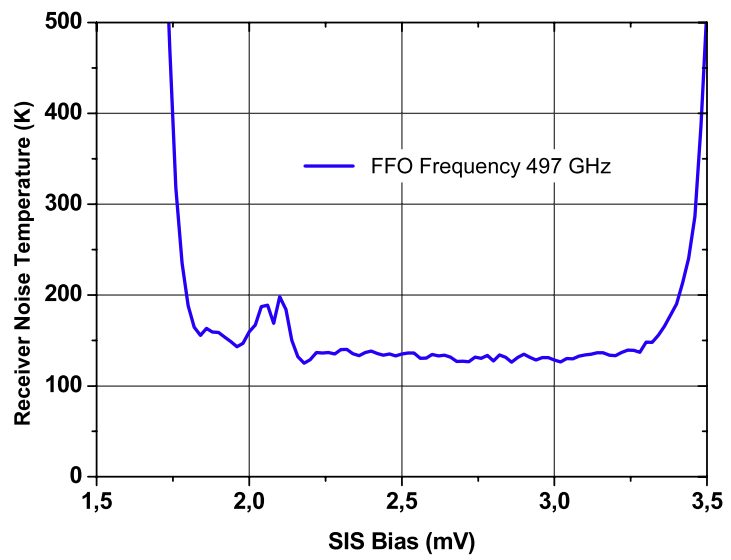

Figure 6. Receiver noise temperature as a function of the SIS bias voltage measured at the FFO frequency $497 \mathrm{GHz}$.

circuitry. The relatively high noise in this band is of no concern for science observations, since this part of the atmospheric spectrum is obscured by a highly saturated water-vapour line rendering it virtually useless for atmospheric science. The noise as a function of IF is fairly flat in the frequency range 4-8 GHz, as can be seen in figure 5. The dependence of the receiver noise temperature on the SIS bias voltage is shown in figure 6 and one can see that for $\mathrm{Nb}-\mathrm{AlN} / \mathrm{NbN}$ circuits there is very wide range of SIS bias voltages where $T_{\mathrm{R}}$ is almost constant.

To ensure stable FFO phase locking, a reasonably good spectral ratio (SR) is needed. This ratio is defined as the ratio between the carrier and the total power emitted by the FFO. The SR value for the given PLL system is unambiguously related to the free-running FFO linewidth [10] and it was found that this linewidth has to be well below $10 \mathrm{MHz}$. It is noted that the SR can be estimated in-flight and therefore the exact FFO line shape can be reconstructed which is of relevance for the data analysis.

The measured free-running linewidth in the frequency band $350-750 \mathrm{GHz}$ ranges from 0.5 to $7 \mathrm{MHz}[8,10,21]$ allowing to phase lock $95 \%-35 \%$ of the emitted FFO power. An example of the free-running (frequency-locked [22]) and 


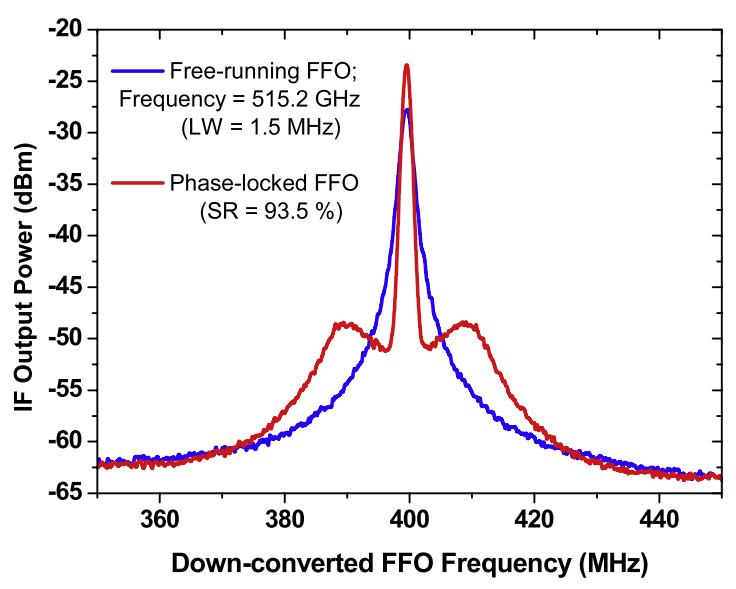

Figure 7. Spectra of the FFO operating at $515.2 \mathrm{GHz}$ (blue curve-frequency locked; red curve-phase locked). Linewidth $(\mathrm{LW})=1.5 \mathrm{MHz}$; signal to noise ratio $(\mathrm{SNR})=36 \mathrm{~dB}$; spectral ration $(\mathrm{SR})=93.5 \%$. Spectra measured with $\mathrm{RBW}=1 \mathrm{MHz}$, $\operatorname{span}=100 \mathrm{MHz}$.

Table 1. Data for the flight SIR at selected TELIS frequencies.

\begin{tabular}{lllll}
\hline $\begin{array}{l}\text { FFO } \\
\begin{array}{l}\text { frequency } \\
(\mathrm{GHz})\end{array}\end{array}$ & $\begin{array}{l}\text { Free-running } \\
\text { LW }(\mathrm{MHz})\end{array}$ & $\begin{array}{l}\text { SNR } \\
(\mathrm{dB})\end{array}$ & $\begin{array}{l}\text { SR } \\
(\%)\end{array}$ & $\begin{array}{l}\text { FFO Ib } \\
(\mathrm{mA})\end{array}$ \\
\hline 495.0 & 1.5 & 32 & 90 & 30.7 \\
496.9 & 1.5 & 37 & 93 & 31.3 \\
515.2 & 1.5 & 36 & 93.5 & 29.2 \\
607.7 & 1.8 & 32.6 & 88.6 & 34 \\
619.1 & 4.6 & 26.8 & 70.3 & 34 \\
\hline
\end{tabular}

phase-locked spectrum of the FFO measured for the flight SIR at $515.25 \mathrm{GHz}$ (one of the selected flight frequencies) is presented in figure 7.

Data for five selected flight frequencies are summarized in table 1. It should be mentioned that the linewidth values are slightly increased due to noise generated by the digital electronics at frequencies of about $1 \mathrm{MHz}$. When the FFO is phase locked this noise is suppressed by the PLL system resulting in a high enough SR.

To investigate the frequency resolution of the receiver, the signal of a solid-state multiplier driven by a synthesizer, was coupled in. The output signal recorded by a spectrum analyser with $30 \mathrm{kHz}$ resolution bandwidth is the convolution of the delta-like function of the synthesizer with the phase-locked signal of the FFO; this measurement indicates a frequency resolution of the SIR receiver better than $100 \mathrm{kHz}$.

The SIR is a complicated device as it contains multiple interactive superconducting elements: a SIS mixer, an FFO, and an HM for the FFO phase locking. Special algorithms and procedures have been developed and tested to facilitate characterization of the SIR at reasonable timescales and for the SIR control during the flight. These routines include:

- fast definition of the FFO operational conditions (both on the Fiske step and in the flux-flow regimes);

- measurements of the free-running FFO linewidth;

- optimization of the LSU and HM parameters;

- optimization of the PLL operation;

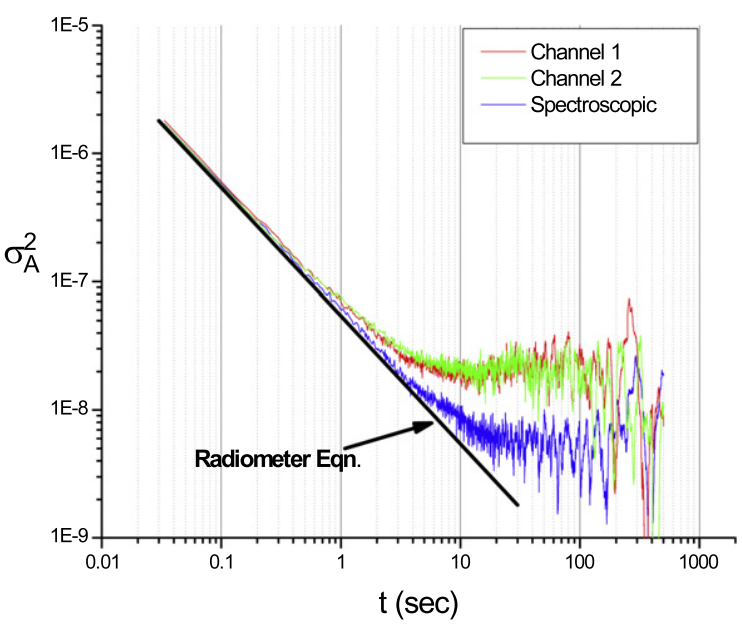

Figure 8. System stability of the SIR channel. FFO is phase locked at $600 \mathrm{GHz}$. The two lines at the top (red and green) represent individual channel variances, the blue line is representative of the spectroscopic variance and the straight black line corresponds to the radiometer equation.

- minimization of the SIR noise temperature;

- setting all predefined SIR parameters in the exact sequence for control during the flight;

- continuous monitoring of the main SIR parameters;

- adjustment (or recovering) of the SIR operational state.

For the TELIS measurement strategy it is important to know whether the timing of limb sounding should depend on the stability of the complete receiver chain. The stability determines the optimum achievable measurement time for a single integration, and thus the required frequency of the calibration cycle. The stability of the complete TELISSIR system has been determined with a noise-fluctuation bandwidth of $17 \mathrm{MHz}$ and the results [3] are presented in figure 8. For the two IF channels that are used to determine the Allan variance it is found that the Allan stability time is about $13.5 \mathrm{~s}$. When the difference of the two channels is taken to determine the Allan variance (this is the so-called spectroscopic, or differential, mode), an Allan stability time of $20 \mathrm{~s}$ is found. This is comparable to stabilities measured for astronomical receivers.

Within TELIS a $1.5 \mathrm{~s}$ integration time per tangent height is used. This is mainly driven by the required integrated signal levels at the autocorrelator input. The stability of the SIR channel therefore poses no constraints on the observing strategy.

\section{Kiruna 2009 campaign}

TELIS had its first successful scientific campaign from Kiruna, North Sweden. The instrument was launched together with the MIPAS instrument on the MIPAS-B2 gondola at $0 \mathrm{~h} 18$, local time, on 11 March 2009. During ascent the SIR channel behaved nominally and already after $30 \mathrm{~min}$ the first spectra were recorded. After $3 \mathrm{~h}$ the first flight ceiling was reached around $35 \mathrm{~km}$. Several night recordings were taken, necessary for background measurements for species with a diurnal cycle 


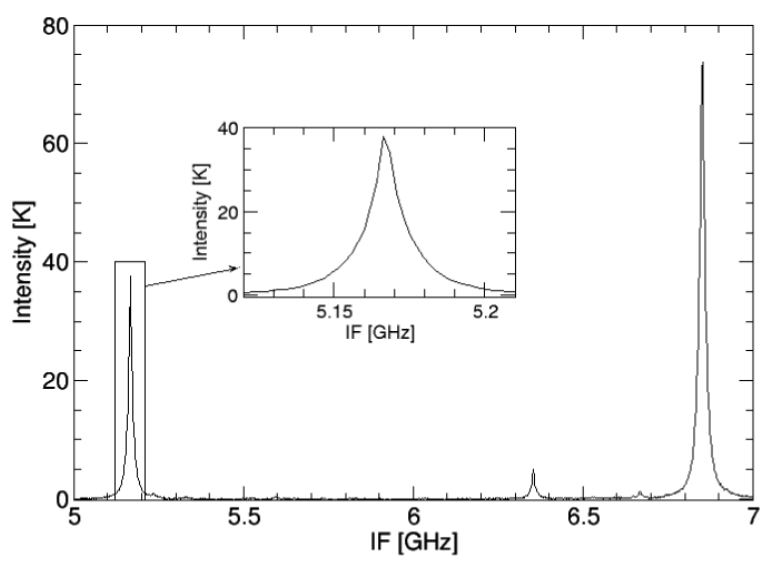

Figure 9. Measured spectra with an LO frequency of $505.600 \mathrm{GHz}$, flight altitude is $35780 \mathrm{~m}$; all visible lines are by $\mathrm{O}_{3}$.

and for instrument calibration. The instrument proved to be stable against the strong temperature variations of the atmosphere during ascent (with ambient temperatures as low as minus $70^{\circ} \mathrm{C}$ ) and during sun-rise.

Due to strong western winds the balloon was lowered to approximately $30 \mathrm{~km}$. The wind direction changed to south east, resulting in a long flight over Finland. During the whole morning the diurnal cycle of various species was monitored until the very end of the flight at $11 \mathrm{~h} 30$. In total several hundred limb sequences have been recorded.

The MIPAS-TELIS balloon system performed nominally during the flight. After parachute landing and recovery the instruments were found to be undamaged, allowing for extra post-flight calibration measurements.

In the early winter of 2009 part of the Arctic vortex was over Northern Scandinavia, which would have allowed for observations on perturbed (ozone) chemistry. At that time, however, launches could not take place due to weather conditions and instrument readiness.

Unfortunately, the vortex did not recover in the remainder of the winter, but at the flight day some remnants were still visible in Northern direction which resulted in slightly enhanced $\mathrm{ClO}$ levels.

\section{Preliminary science results}

The science goals of the campaign in March 2009 from Kiruna, North Sweden, were threefold: investigation of the stratospheric hydrological cycle by measurements of isotopic water, catalytic ozone destruction by chlorine chemistry, and the bromine content of the stratosphere. Data presented in figure 9 prove the capabilities of the TELIS-SIR channel for high resolution spectroscopy. In this case the FFO frequency is tuned to $505.6 \mathrm{GHz}$, the telescope is $6^{\circ}$ up-looking and the gondola altitude is $35.78 \mathrm{~km}$. The width of the ozone line is measured to be about $10 \mathrm{MHz}$, as expected (see inset in figure 9).

The data reduction is on-going, but the first preliminary science results are presented for $\mathrm{HCl}$. Chlorine ozone destruction peaks in the arctic winter and/or spring when the

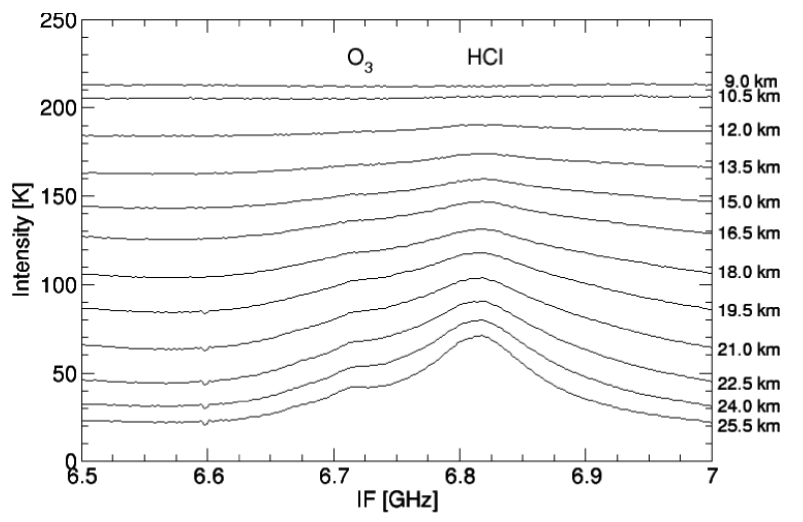

Figure 10. Measured spectra with an LO frequency of $619.100 \mathrm{GHz}$. The most pronounced feature is by $\mathrm{HCl}$. At the right-hand side the altitude is given for which the particular viewing geometry is most sensitive.

so-called polar vortex breaks up. During this event the $\mathrm{ClO}$ radical, responsible for catalytic ozone destruction, becomes available in huge amounts. However, chlorine is also stored in non-reactive reservoir species of which $\mathrm{HCl}$ is an important member. The amount of $\mathrm{HCl}$ in the stratosphere is a measure of the total non-active $\mathrm{Cl}$ content and is as such an important species to monitor in ozone chemistry studies.

In figure 10 measured spectra are shown. The $\mathrm{HCl}$ line is well pronounced for several viewing geometries. A viewing geometry is determined by the flight altitude of the balloon platform and the tilt of the telescope. Each geometry results in different altitude sensitivities and effectively probes different parts of the atmosphere. The altitudes mentioned in figure 10 refer to the lowest probed altitude for a certain viewing geometry and generally corresponds to the altitude for which the measurement is mostly sensitive.

The radiometric calibration is done in a straightforward way by taking the closest calibration black body and cold sky spectra without optimization. Sideband ratios as well as a radiometric error of the autocorrelator are not considered. The radiometric uncertainty now estimated to be larger than $20 \%$. Furthermore, the field of view function of the antenna beam was not yet considered in the retrieval. Due to these uncertainties, the spectra are first normalized against a background intensity before retrieval is conducted.

Full level 1 data processing including characterization is in progress.

Retrieving a vertical $\mathrm{HCl}$ profile from these spectra is a socalled ill-posed problem. This means that a whole ensemble of profiles will reproduce the measurement within noise-limits. Many approaches have been developed to solve this kind of problems and in this study the Tikhonov regularization method has been adopted [23-25]. This method is basically a least squares fit in conjunction with an additional constraint that suppresses noise contributions of the measurement to the retrieved vertical profile. The method does not require $a$ priori knowledge, but relies fully on the information content of the measurement.

In figures 11 and 12 the $\mathrm{HCl}$ retrieval results are depicted. In figure 11 the solid black line is the retrieved vertical profile 


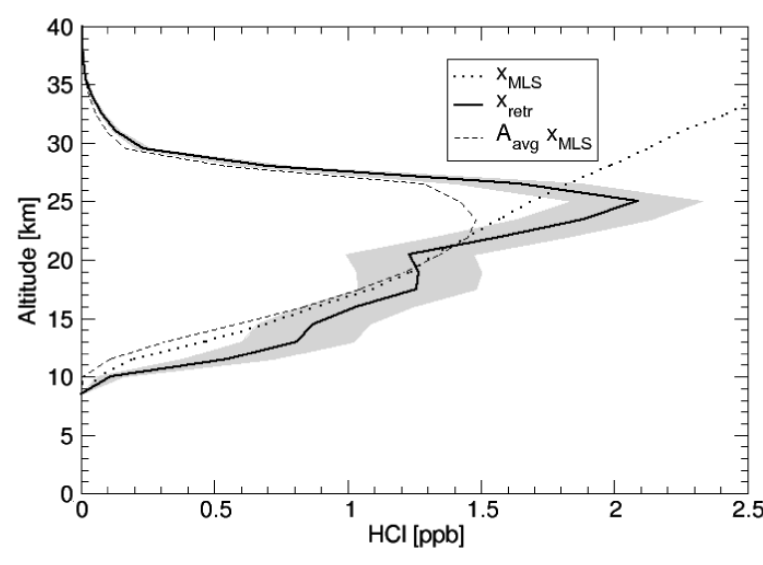

Figure 11. The retrieved (solid) vertical $\mathrm{HCl}$ profile with the retrieval error indicated by the grey area. The dotted and dashed lines are respectively the retrieved profile by the Aura/MLS satellite and its mapping by the averaging kernel.

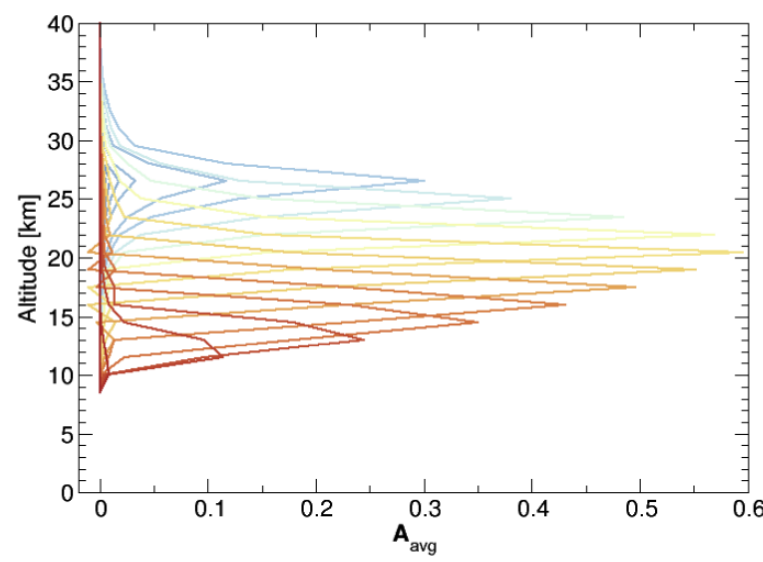

Figure 12. The averaging kernel maps the true atmospheric profile to the retrieved profile.

and the grey area indicates the retrieval error. It can be seen that the profile collapses above $27 \mathrm{~km}$. This should not be interpreted as a strongly reduced $\mathrm{HCl}$ content at these altitudes but indicates the limits of the information content in the measurements. This can also be seen in figure 12 where the averaging kernel is depicted. The averaging kernel $\mathbf{A}_{\text {avg }}$ maps the true vertical profile to the retrieved profile by $\boldsymbol{x}_{\text {retr }}=\mathbf{A}_{\text {avg }} \boldsymbol{x}_{\text {true }}+\varepsilon$, where $\boldsymbol{x}$ represents the vertical profiles and $\varepsilon$ the retrieval error. If $\mathbf{A}_{\text {avg }}$ would be the identity matrix, then all information of the true atmospheric profile would be captured by the retrieved profile. In general, the retrieved profile is a weighted average of this true profile over a certain altitude range. The narrower the peaks are in the averaging kernel, the better the vertical spatial resolution is. For altitudes beyond $27 \mathrm{~km}$, the kernel not only broadens, but also lowers in amplitude, resulting in the collapse of the retrieved vector. For details on the retrieval procedure and on how the averaging kernel is obtained we refer to [26].

To validate the quality of the retrieved profile, the profile is compared against a reference $\mathrm{HCl}$ profile measured co-located in time and space by another instrument. In figure 11 the dotted line corresponds to the $\mathrm{HCl}$ profile as measured by the
Aura/MLS satellite instrument. To compare the two profiles, the reference $\mathrm{HCl}$ profile needs to be mapped by the averaging kernel and results in the dashed line in figure 11. It can be seen that this line is not fully covered by the grey area, but the overall shape is similar and peaks at the same altitude. The slope of the TELIS and reference profiles are very similar for altitudes below $25 \mathrm{~km}$ which is the range where the TELIS measurement is most sensitive.

It should be noted that in the retrieval procedure a sideband ratio (SBR) of 1.0 is assumed. This means that the gains of the upper and lower sidebands are taken identical which is not necessarily the case. The actual sideband ratio is to be determined in a laboratory characterization campaign. A depart of the SBR from 1.0 will result in an overall shift of the retrieved profile, either improving or deteriorating the coincidence with the reference profile.

This result is very preliminary and the retrievals and instrument characterization is still on-going. However, this result looks promising for the future data analysis of TELIS.

\section{Conclusion}

The capability of the superconducting integrated receiver for high resolution atmospheric spectroscopy has been successfully proven with a first scientific balloon flight in March 2009 from Kiruna, North Sweden. During the $11 \mathrm{~h}$ mission, phase-locked SIR operation and frequency switching in the $450-700 \mathrm{GHz}$ frequency range has been realized. An intrinsic spectral resolution of the SIR well below $1 \mathrm{MHz}$ has been confirmed by CW signal measurements. An uncorrected double-sideband noise temperature below $120 \mathrm{~K}$ has been measured for the SIR when operated with a phase-locked FFO and an intermediate frequency bandwidth of 4-8 GHz. To ensure remote operation of the phase-locked SIR several software procedures for automatic control have been developed and tested. The first tentative $\mathrm{HCl}$ profile has been presented and its quality looks promising for future data reduction.

\section{Acknowledgments}

The authors thank colleagues at DLR, IPM, IREE and SRON for help and assistance in the SIR channel design and characterization: J Barkhof, A Baryshev, J Kooi, O Koryukin, A Pankratov, S Pripolzin, O Pylypenko, M Romanini, S Shitov and V Vaks; also T de Graauw and W Wild are acknowledged for their support of this work.

\section{References}

[1] Suttiwong N, Birk M, Wagner G, Krocka M, Wittkamp M, Haschberger P, Vogt P and Geiger F 2009 Proc. 19th ESA Symp. on European Rocket and Balloon Programmes and Related Research (Bad Reichenhall) at press

[2] Hoogeveen R W M, Yagoubov P A, de Lange A, Selig A M, Koshelets V P, Ellison B N and Birk M 2005 Proc. SPIE $\mathbf{5 9 7 8} 440$

[3] Hoogeveen R W M, Yagoubov P A, de Lange G, de Lange A, Koshelets V, Birk M and Ellison B 2007 Proc. SPIE 6744 $67441 \mathrm{U}$ 
[4] Friedl-Vallon F, Maucher G, Seefeldner M, Trieschmann O, Kleinert A, Lengel A, Keim C, Oelhaf H and Fischer H 2004 Appl. Opt. 433335

[5] Koshelets V P, Shitov S V, Filippenko L V, Baryshev A M, Golstein H, de Graauw T, Luinge W, Schaeffer $\mathrm{H}$ and van de Stadt H 1996 Appl. Phys. Lett. 681273

[6] Koshelets V P and Shitov S V 2000 Supercond. Sci. Technol. 13 R53

[7] Yagoubov P, Hoogeveen R, Torgashin M, Khudchenko A, Koshelets V, Suttiwong N, Wagner G and Birk M 2006 Proc. ISSTT p 338

[8] Koshelets V P, Ermakov A B, Filippenko L V, Khudchenko A V, Kiselev O S, Sobolev A S, Torgashin M Yu, Yagoubov P A, Hoogeveen R W M and Wild W 2007 IEEE Trans. Appl. Supercond. 17336

[9] Nagatsuma T, Enpuku K, Irie F and Yoshida K 1983 J. Appl. Phys. 543302

Nagatsuma T, Enpuku K, Yoshida K and Irie F 1984 J. Appl. Phys. 563284

Nagatsuma T, Enpuku K, Sueoka K, Yoshida K and Irie F 1985 J. Appl. Phys. 58441

Qin J, Enpuku K and Yoshida K 1988 J. Appl. Phys 631130

[10] Koshelets V P, Dmitriev P N, Ermakov A B, Sobolev A S, Torgashin M Yu, Kurin V V, Pankratov A L and Mygind J 2005 IEEE Trans. Appl. Supercond. 15964

[11] Mehdi I 2004 Proc. SPIE 5498103

[12] Livesey N, Santee M, Stek P, Waters J, Levelt P, Veefkind P, Kumer J and Roche A 2007 EOS Trans. Am. Geophys. Union 88 A12A-08 (Fall Meet. Suppl.)

[13] Torgashin M Yu, Koshelets V P, Dmitriev P N, Ermakov A B, Filippenko L V and Yagoubov P A 2007 IEEE Trans. Appl. Supercond. 17379

[14] Koshelets V P, Shitov S V, Shchukin A V, Filippenko L V, Dmitriev P N, Vaks V L, Mygind J, Baryshev A B,
Luinge W and Golstein H 1999 IEEE Trans. Appl. Supercond. 94133

[15] Yagoubov P, van de Stadt H, Hoogeveen R, Koshelets V, Birk M and Murk A 2005 Proc. 28th ESA Antenna Workshop on Space Antenna Systems and Technologies (Noordwijk) vol 2, p 763

[16] Yagoubov P A, Vreeling W-J, van de Stadt H, Hoogeveen R W M, Koryukin O V, Koshelets V P, Pylypenko O M and Murk A 2005 Proc. 16th Int. Conf. on Space Terahertz Technology (Gothenburg) p 438

[17] Murk A, Yagoubov P, Mair U, Birk M, Wagner G, van de Stadt H, Hoogeveen R and Kämpfer N 2005 Proc. 28th ESA Antenna Workshop on Space Antenna Systems and Technologies (Noordwijk) p 757

[18] Ellison B N, Moyna B P, Matheson D N, Jones A, Claude S M X, Mann C, Kerridge B J, Siddans R, Munro R and Reburn W J 1998 Proc. 2nd ESA Workshop on Millimetre Wave Technology and Applications (Espoo)

[19] Cherednichenko S, Drakinskiy V, Berg T, Khosropanah P and Kollberg E 2008 Rev. Sci. Instrum. 79034501

[20] Emrich A, Andersson S and Knis M 2006 Proc. Joint 31st Int. Conf. on Infrared Millimeter Waves and 14th Int. Conf. on Teraherz Electronics (Shanghai) p 314

[21] Koshelets V P, Shitov S V, Ermakov A B, Koryukin O V, Filippenko L V, Khudchenko A V, Torgashin M Yu, Yagoubov P, Hoogeveen R and Pylypenko O M 2005 IEEE Trans. Appl. Supercond. 15960

[22] Koshelets V P, Shitov S V, Shchukin A V, Filippenko L V and Mygind J 1996 Appl. Phys. Lett. 69699

[23] Tikhonov A 1963 Dokl. Akad. Nauk SSSR 151501

[24] Twomey S 1963 J. Assoc. Comput. Mach. 1097

[25] Phillips P 1962 J. Assoc. Comput. Mach. 984

[26] de Lange A, Landgraf J and Hoogeveen R 2009 Atmos. Meas. Technol. 2423 